\title{
Surface plasmon enhanced photocurrent in thin GaAs solar cells
}

Keisuke Nakayama, Katsuaki Tanabe, Harry A. Atwater

Keisuke Nakayama, Katsuaki Tanabe, Harry A. Atwater, "Surface plasmon enhanced photocurrent in thin GaAs solar cells," Proc. SPIE 7047, Nanoscale Photonic and Cell Technologies for Photovoltaics, 704708 (11 September 2008); doi: $10.1117 / 12.795469$

SPIE Event: Solar Energy + Applications, 2008, San Diego, California, United States 


\title{
Surface plasmon enhanced photocurrent in thin GaAs solar cells
}

\author{
Keisuke Nakayama*, Katsuaki Tanabe, Harry A. Atwater** \\ Thomas J. Watson Laboratory of Applied Physics, California Institute of Technology, Pasadena, \\ CA, USA 91125
}

\begin{abstract}
We report fabrication of size-controlled plasmonic nanoparticle arrays by which optically thin GaAs single junction solar cells are decorated. Ordered Ag and Al nanoparticles with average diameters of 60-150 nm and interparticle spacings of 100-300 $\mathrm{nm}$ were templated onto the window layers of the GaAs solar cells using nanoporous anodic aluminum oxide membrane templates. Near the surface plasmon resonances, $60 \mathrm{~nm}$-diameter Ag and Al nanoparticles serve as lightabsorbers so that non-radiative surface plasmon resonances reduce the photocurrent of the cells, which is improved by increasing the nanoparticle size. Photocurrent enhancements are seen at wavelengths longer than surface plasmon resonance which is maximized near the band gap edge of GaAs. These enhancements can be attributed to the increased optical path in the photovoltaic layers resulting from multi-angle scattering by the nanoparticles, while high scattering efficiency nanoparticles in turn increase the back scattering light out of the cell reducing the photocurrent.
\end{abstract}

Keywords: plasmonic nanoparticle, Ag, Al, anodic aluminum oxide, GaAs solar cell

\section{INTRODUCTION}

The large optical extinction of plasmonic nanoparticles near the surface plasmon resonance has potential to enhance the solar light harvesting for a variety of applications. In recent years, a close coupling of plasmonic nanoparticles to absorbing semiconductor has been utilized to enhance absorption in ultrathin film solar cells. ${ }^{[1-5]}$ The extinction of the nanoparticles is defined as the sum of the scattering and absorption. For spherical nanoparticles in the quasistatic limit, the scattering and absorption efficiencies are:

$$
Q_{a b s} \equiv 4 \chi \operatorname{Im}\left(\frac{\varepsilon_{1}-\varepsilon_{m}}{\varepsilon_{1}+2 \varepsilon_{m}}\right), \quad Q_{s c a} \equiv \frac{8}{3} \chi^{4}\left|\frac{\varepsilon_{1}-\varepsilon_{m}}{\varepsilon_{1}+2 \varepsilon_{m}}\right|^{2},
$$

where the size parameter $\chi$ is the circumference of the nanoparticle divided by wavelength, and $\varepsilon_{m}$ and $\varepsilon_{1}$ are the permittivity of the medium and metal, respectively. ${ }^{[6]}$ The radiation efficiency $\eta_{\text {rad }}$ is given by $\eta_{\text {rad }} \equiv Q_{s c a} / Q_{e x t}=$ $Q_{s c a} /\left(Q_{a b s}+Q_{s c a}\right)$. From the perspective of photovoltaic conversion, plasmon resonant absorption represents an unwanted loss process, but off-resonant enhanced scattering can yield increases in absorption and photocurrent collection. Since the optical property of the plasmonic nanoparticles sensitively depends on nanoparticle size and shape, proper morphological design of the nanoparticles is a requirement for achieving high efficiency plasmonic solar cells.

In this work, dense arrays of metal nanoparticles were templated using nanoporous anodic aluminum oxide (AAO) membranes onto optically thin GaAs solar cells. Anodic aluminum oxide has been widely used as a template for nanostructure formation since the nanopore diameter, density, and length can be controlled simply by varying the anodization and etching conditions. ${ }^{[7-10]}$ Hemispherical $\mathrm{Ag}$ and $\mathrm{Al}$ nanoparticles with average diameters of $60 \mathrm{~nm}, 110$ $\mathrm{nm}$, and $150 \mathrm{~nm}$ were formed onto the window layers of the GaAs solar cells and nanoparticle size effect on the photocurrent of the cells was investigated. Both $\mathrm{Ag}$ and $\mathrm{Al}$ nanoparticles had similar effects on the photocurrent responses of the GaAs cells; increasing the nanoparticle size caused the dominant scattering rather than the absorption near the surface plasmon resonances. Photocurrent was enhanced at wavelengths longer than surface plasmon resonances and the enhancement was maximized near the band gap of GaAs, which was quantitatively described by a calculated absorption in which multi-angle light scattering by the nanoparticles were taken into account.

*Also at Nippon Oil Corporation, Yokohama, Japan 231-0815; **haa@caltech.edu; phone 1 626 395-2197; fax 1626 844-9320

Nanoscale Photonic and Cell Technologies for Photovoltaics, edited by Loucas Tsakalakos, Proc. of SPIE Vol. 7047, 704708, (2008) · 0277-786X/08/\$18 · doi: 10.1117/12.795469 


\section{EXPERIMENT}

The structure of a GaAs solar cell used in this work is shown in Fig. 1. The cell was grown on (100) $n$-GaAs substrate by metalorganic chemical vapor deposition with $\mathrm{Zn}$ as the $p$-type dopant and $\mathrm{Si}$ as the $n$-type dopant. To clarify the photocurrent enhancement by plasmonic nanoparticles, optically thin GaAs solar cell, in which the photovoltaic layers are much thinner than the absorption length, was utilized. Compared to the high performance GaAs cells, the thickness of the $p$ - $n$ GaAs photovoltaic layers is less than $10 \%$ with the depletion layer thickness unchanged ${ }^{[11]}$ In addition, high band gap back surface field (BSF) layer of $n-\mathrm{Al}_{0.8} \mathrm{Ga}_{0.2} \mathrm{As}$ is formed to avoid carrier generation in the BSF layer. ${ }^{[12]}$ The calculated absorption spectrum shows that half of the sunlight above the band gap of GaAs is absorbed by the $p$ $\mathrm{Al}_{0.8} \mathrm{Ga}_{0.2} \mathrm{As}$ window and $p-n$ GaAs photovoltaic layers. The remaining light transmitted through the layers is absorbed by the buffer layer and the substrate without carrier collection.
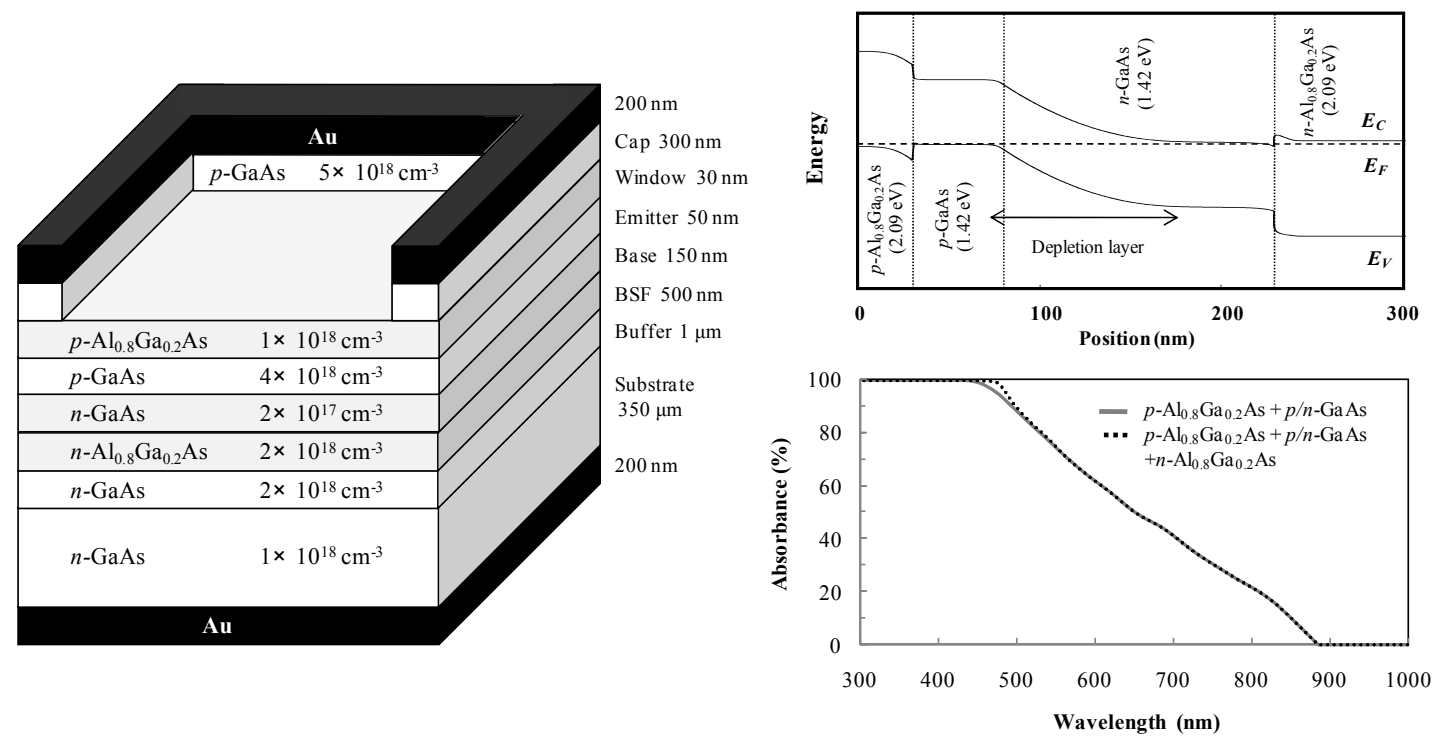

Fig. 1. Optically thin GaAs solar cell with $\mathrm{Al}_{0.8} \mathrm{Ga}_{0.2} \mathrm{As}$ window and BSF layers. 100nm-thick depletion layer is located at the center of the $200 \mathrm{~nm}$-thick GaAs photovoltaic layers. Photocurrent generates in the top three layers evidenced by the calculated absorption spectra.

After both front and bottom Au contacts were formed, the GaAs cap layer was selectively etched in 4: 1 citric acid $(50 \%): \mathrm{H}_{2} \mathrm{O}_{2}(30 \%)$ solution, ${ }^{[13]}$ Then ordered array of metal nanoparticles was directly deposited onto the window layer of the cell trough an AAO template by thermal evaporation. AAO templates having straight nanopores were fabricated through a two-step anodization of $4 \mathrm{~N}$-grade aluminum sheet. Anodization was carried out at $10{ }^{\circ} \mathrm{C}$ in three different conditions; (1) at $40 \mathrm{~V}$ in a $0.3 \mathrm{molL}^{-1}$ oxalic acid solution (referred to as " $40 \mathrm{~V}-\mathrm{AAO}$ "), (2) at $80 \mathrm{~V}$ in a mixture of 0.3 molL ${ }^{-1}$ oxalic acid and $0.3 \mathrm{molL}^{-1}$ malonic acid solutions (referred to as " $80 \mathrm{~V}-\mathrm{AAO}$ "), and (3) at $120 \mathrm{~V}$ in a $0.3 \mathrm{molL}^{-1}$ malonic acid solution (referred to as "120V-AAO"). ${ }^{[14]}$ The thickness of the AAO layer formed on aluminum sheet was controlled around $700 \mathrm{~nm}$. Afterwards, the aluminum sheet was selectively removed in saturated iodine solution in methanol, and the bottom layer of the AAO was chemically etched in $5 \%$ dilute solution of phosphoric acid (85\%) at 30 ${ }^{\circ} \mathrm{C}$ to obtain a through-hole membrane. The through-hole AAO template about $4 \times 4 \mathrm{~mm}^{2}$ in size was then placed directly onto the window layer of the cell and $\mathrm{Ag}$ or $\mathrm{Al}$ was thermally evaporated, followed by removing the template and annealing at $200{ }^{\circ} \mathrm{C}$ for 30 minutes in $5 \% \mathrm{H}_{2}$ diluted by $\mathrm{N}_{2}$. The height of the metal nanoparticles was controlled simply with the deposition thickness in the thermal evaporation process, which was $35 \mathrm{~nm}$ through $40 \mathrm{~V}-\mathrm{AAO}, 55 \mathrm{~nm}$ through $80 \mathrm{~V}-\mathrm{AAO}$, and $75 \mathrm{~nm}$ through $120 \mathrm{~V}-\mathrm{AAO}$, respectively.

The scanning electron microscopy (SEM) images were taken using an S-4100 scanning electron microscope (Hitachi) at $30 \mathrm{kV}$. The extinction spectrum was characterized using an SE850 elipsometer (Sentech) for the nanoparticle array formed on glass substrate through the AAO template. Spectral response measurements were carried out by shining 
monochromated light from a $300 \mathrm{~W}$ Xenon lamp. The current-voltage $(I-V)$ characteristics were measured under one sun illumination (AM1.5, $100 \mathrm{mWcm}^{-2}$ ) using a solar simulator. The exposed area of all the cells was limited to $3 \mathrm{~mm} \times 3$ $\mathrm{mm}$ by using a metal-mask.

\section{RESULTS AND DISCUSSION}

\subsection{Characterization of metal nanoparticle arrays}

Figure 2 shows SEM images of the AAO templates and corresponding Ag and Al nanoparticle arrays formed on the GaAs solar cells. The AAO templates possessed the nanopores with $60 \mathrm{~nm}$-diameter and $1.0 \times 10^{10} \mathrm{~cm}^{-2}-\mathrm{density}$ for $40 \mathrm{~V}$ AAO, with $110 \mathrm{~nm}$-diameter and $3.3 \times 10^{9} \mathrm{~cm}^{-2}$-density for $80 \mathrm{~V}$-AAO, and with $150 \mathrm{~nm}$-diameter and $1.8 \times 10^{9} \mathrm{~cm}^{-2}-\mathrm{density}$ for $120 \mathrm{~V}-\mathrm{AAO}$. Evaporation of metal through these templates resulted in formation of metal nanoparticle arrays on top of GaAs solar cells with the same diameters and interspacings as the nanopores in the AAO templates. Ag and Al nanoparticles were uniformly deposited in the entire area where the AAO templates were placed.
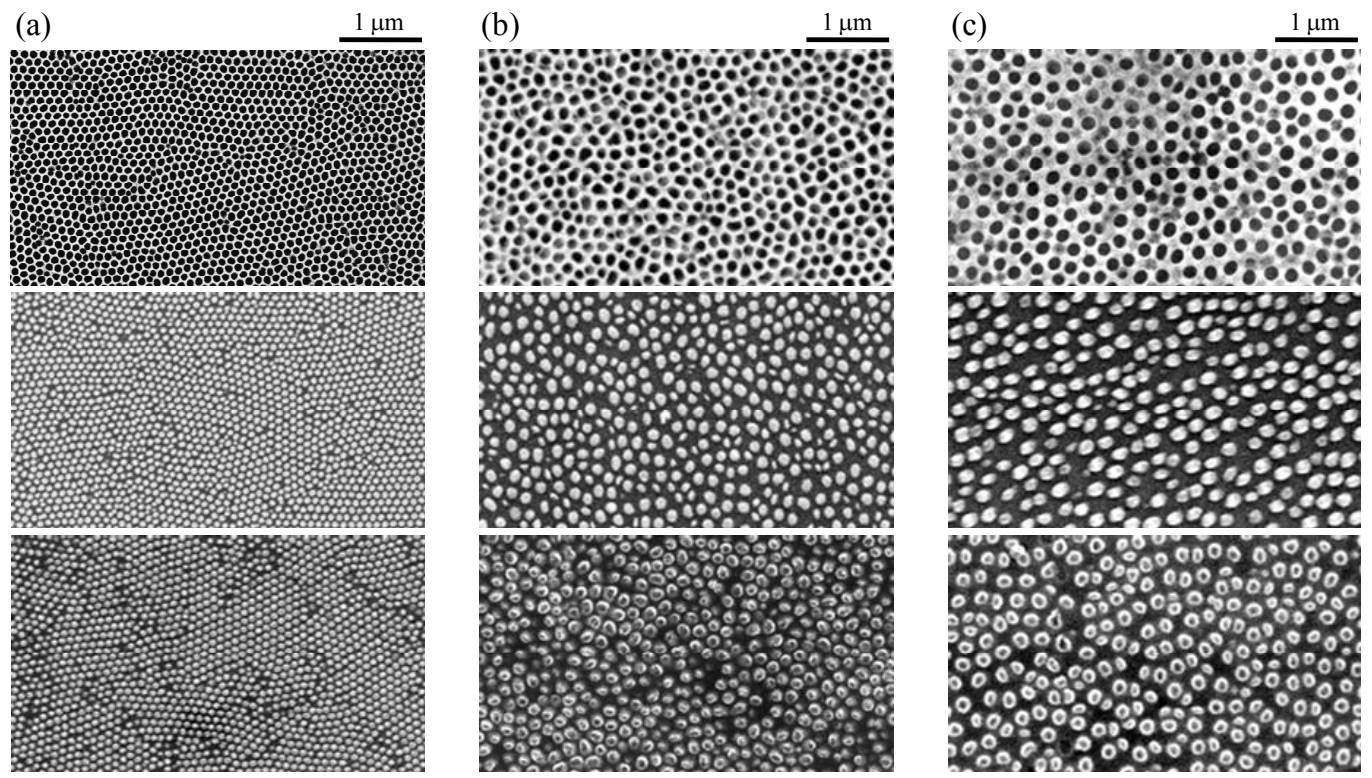

Fig. 2. SEM images of AAO templates (top images) and as-deposited Ag (middle images) and Al (bottom images) nanoparticle arrays formed on the GaAs solar cells through the templates; (a) 40V-AAO, (b) 80V-AAO and (c) 120VAAO.

The morphology of $\mathrm{Al}$ nanoparticles remained unchanged after annealing at $200{ }^{\circ} \mathrm{C}$ for 30 minutes, while annealing the $\mathrm{Ag}$ nanoparticles induced the structural changes to more hemispherical shapes relative to those immediately after deposition as shown in Fig. 3. Annealing the Ag nanoparticles at temperatures well below the melting point of silver caused surface melting migration of Ag resulting in the restructured Ag nanoparticles with thermally stabilized shape, ${ }^{[15]}$ while surface aluminum oxide layers might prevent the structural changes in $\mathrm{Al}$ cases. These structural changes of $\mathrm{Ag}$ nanoparticles are also seen in the extinction spectra for the nanoparticles formed on glass substrates [Fig. 4]. In the spectra for as-deposited $\mathrm{Ag}$ and $\mathrm{Al}$ nanoparticles, the peaks are clearly visible at wavelengths corresponding to the surface plasmon resonance; $450 \mathrm{~nm}(40 \mathrm{~V}-\mathrm{AAO}), 500 \mathrm{~nm}(80 \mathrm{~V}-\mathrm{AAO}), 520 \mathrm{~nm}$ (120V-AAO) for Ag nanoparticles and $350 \mathrm{~nm}(40 \mathrm{~V}-\mathrm{AAO}), 400 \mathrm{~nm}(80 \mathrm{~V}-\mathrm{AAO}), 450 \mathrm{~nm}$ (120V-AAO) for Al nanoparticles. The absorpsion by the $\mathrm{Ag}$ nanoparticles at $360 \mathrm{~nm}$ is related to electronic interband transitions in Ag. ${ }^{[16]}$ Annealing the Ag nanoparticles yielded the blue-shifted surface plasmon resonance, which can be understood qualitatively based on improvements in the symmetry of the nanoparticle shape ${ }^{[6,17]}$ while annealing had no effect in the spectra for Al nanoparticles. In both $\mathrm{Al}$ and Ag cases, the surface plasmon resonances red-shifted and grew broader as the nanoparticle sizes are increased. 
Considering the similar shape and aspect-ratio of the series of nanoparticles, the red-shifted peaks are chiefly attributed to the increase in the nanoparticle size, which are known to cause the shifts of the surface plasmon resonance to longer wavelengths. ${ }^{[3,6]}$ The broader peaks of larger nanoparticles imply that scattering rather than absorption dominates optical extinction in the larger nanoparticles.
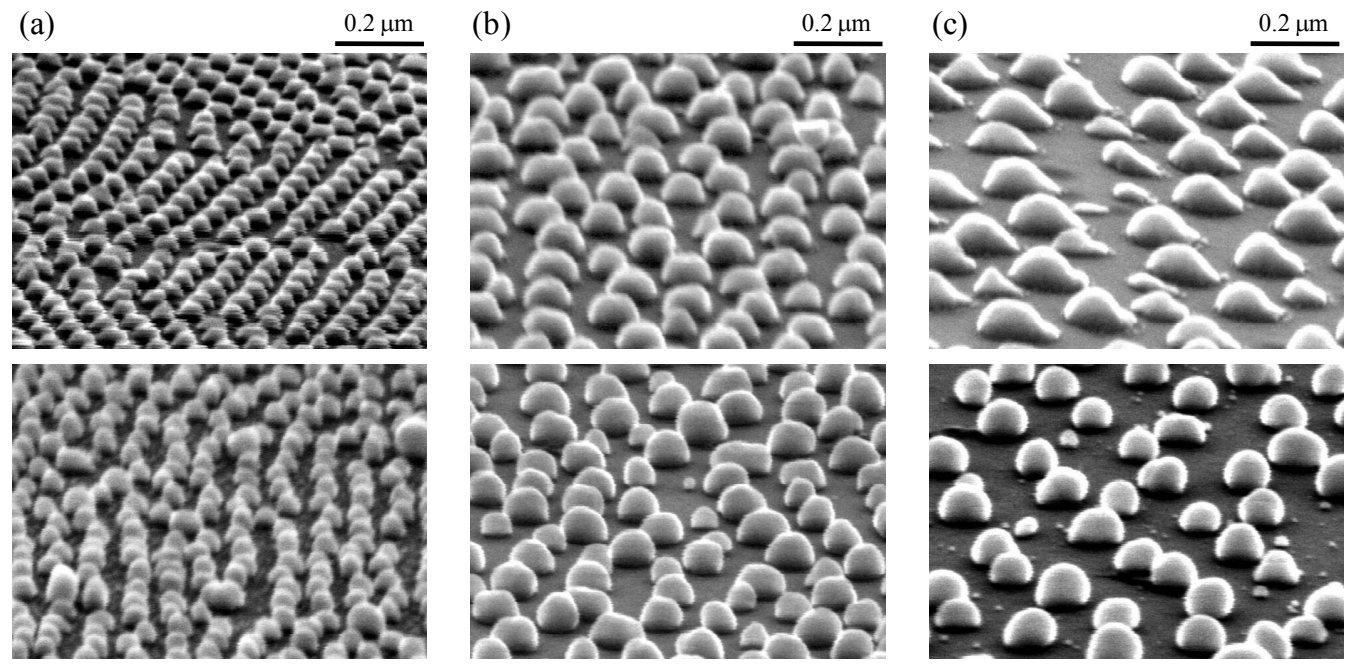

Fig. 3. SEM images of Ag nanparticle arrays through (a) 40V-AAO, (b) 80V-AAO, and (c) 120V-AAO before (top images) and after (bottom images) annealing at $200{ }^{\circ} \mathrm{C}$ for $30 \mathrm{~min}$. The images were taken from the angle of $75^{\circ}$.

(a)

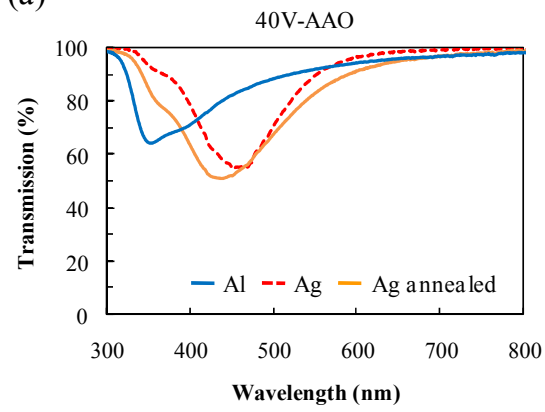

(b)

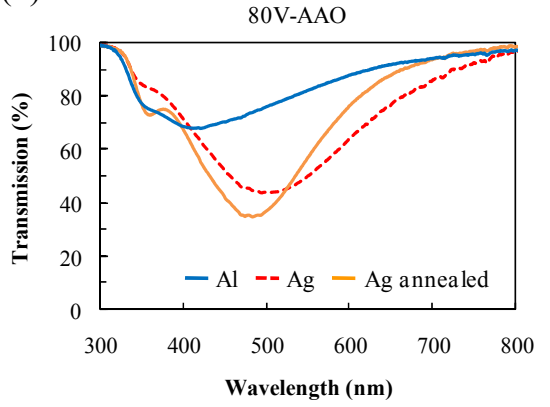

(c)

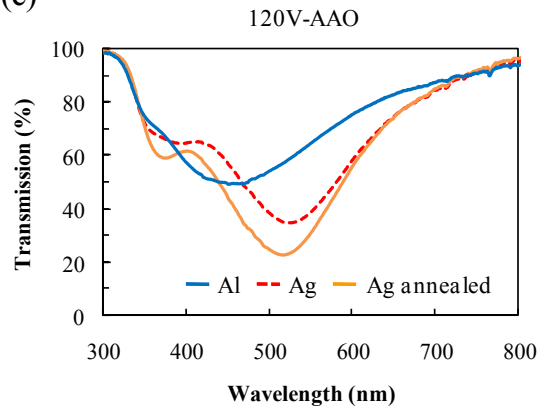

Fig. 4. Extinction spectra for the plasmonic nanoparticle arrays formed on glass substrates through (a) 40V-AAO, (b) 80V$\mathrm{AAO}$, and (c) 120V-AAO.

\subsection{Photocurrent enhancements of the GaAs solar cells with metal nanoparticle arrays}

The external quantum efficiency (EQE) for a reference GaAs solar cell (i.e., without metal nanoparticle array on top) is shown in the inset of Fig. 5(a), which is in good agreement with the calculated absorption in Fig. 1. In order to examine the photocurrent responses clearly, the EQEs of the nanoparticle-decorated GaAs solar cells are normalized by that of the reference cell [Fig. 5(a)]. Annealing the Ag nanoparticles significantly improved the EQEs in the wide range of wavelengths, while similar photocurrent responses before and after annealing were observed in $\mathrm{Al}$ cases. When annealed Ag nanoparticles through 40V-AAO are placed on the GaAs solar cell, the small nanoparticles serve as light-absorbers rather than light-scatters so that non-radiative surface plasmon resonance significantly reduces the incident light into the cell and the photocurrent. At wavelengths longer than the surface plasmon resonance, the normalized EQE is gradually increased and photocurrent enhancement relative to the reference cell is seen at the wavelengths below $660 \mathrm{~nm}$ with the maximum enhancement near the band gap edge of GaAs. At the cell with the medium Ag nanoparticles through $80 \mathrm{~V}$ - 
$\mathrm{AAO}$, the drop in the EQE at surface plasmon resonance is smaller and photocurrent enhancement is seen at the wavelengths below $600 \mathrm{~nm}$ due to the improved radiation efficiency of the nanoparticles. However, the cell with the large Ag nanoparticles through 120V-AAO shows the slightly decreased photocurrent response over a wide range of wavelengths relative to the reference cell. This response implies that the high scattering efficiency nanoparticles in turn increase the back-scattering light out of the cell reducing the photocurrent. The Al nanoparticles have similar effects on the photocurrent responses of the GaAs solar cells. The small Al nanoparticles also serve as light-absorbers, while the blue-shifted surface plasmon resonance improves the EQE relative to the small Ag nanoparticle-decorated cell. The decreased EQEs of the medium and large Al nanoparticle cells relative to the Ag nanoparticle cells indicate that more amount of the incident light is back-scattered by the Al nanoparticles.

In order to understand the role of the metal nanoparticle arrays on the GaAs solar cells more thoroughly, a simple model to represent the absorption enhancement in the photovoltaic layers with plasmonic nanoparticle arrays on top have been developed as follows. For simplification, we considered only the GaAs photovoltaic layers neglecting $\operatorname{the} \mathrm{Al}_{0.8} \mathrm{Ga}_{0.2} \mathrm{As}$ window layer due to the similar refractive indices of $\mathrm{GaAs}_{\text {and }} \mathrm{Al}_{0.8} \mathrm{Ga}_{0.2} \mathrm{As}$. When GaAs layers with thickness of $L$ and absorption constant of $\alpha(\lambda)$ at free space wavelength $\lambda$ absorb the incident light scattered by plasmonic nanoparticles at an angle $\theta$ with respect to the normal, the absorption fraction for the scattered light is:

$$
A_{\theta}(\lambda)=\int_{0}^{\pi / 2} \frac{1+\cos ^{2} \theta}{\int_{0}^{\pi}\left(1+\cos ^{2} \theta\right) d \theta}\left\{1-\exp \left(-\alpha(\lambda) \frac{L}{\cos \theta}\right)\right\} d \theta,
$$

accounting that the optical path in the GaAs layers is increased from $L$ into $L / \cos \theta$. The total absorption fraction for the GaAs layers with plasmonic nanoparticle array with a surface coverage $\xi$ is:

$$
A_{\text {tot }}(\lambda)=\xi Q_{\text {ext }}(\lambda) \eta_{\text {rad }}(\lambda) A_{\theta}(\lambda)+\left(1-\xi Q_{\text {ext }}(\lambda)\right)(1-R(\lambda)) A_{0}(\lambda) \quad\left(\xi Q_{\text {ext }}<1\right) .
$$

The surface coverage $\xi$ was determined from SEM images. Values for $Q_{e x t}$ and $\eta_{\text {rad }}$ were calculated for oblate ellipsoidal nanoparticles with a minor axis parallel to the normal in the quasistatic limit using an effective medium approximation for the complex dielectric function of the surrounding medium. We assumed that the structural change of $\mathrm{Ag}$ nanoparticles after annealing makes the effective nanoparticle central position lower to better fit the experimental results. The wavelength-dependent complex dielectric functions of metal and GaAs were obtained from Reference 18. Particularly for $\mathrm{Al}$ nanoparticles, $Q_{\text {ext }}$ and $\eta_{\text {rad }}$ were calculated for concentric $\mathrm{Al}_{-} \mathrm{Al}_{2} \mathrm{O}_{3}$ core-shell ellipsoidal nanoparticles with an $\mathrm{Al}_{2} \mathrm{O}_{3}$ shell thickness of $4 \mathrm{~nm}$ accounting for surface oxidation of $\mathrm{Al}$ nanoparticles in the atmosphere ${ }^{[19,20]}$ The reflectivity $R$ at the air/GaAs interface is also accounted since the used GaAs cells had no antireflective coating. Assuming normal incidence of light into a GaAs layer, the wavelength-dependent reflectivity is written as:

$$
R(\lambda) \equiv \frac{I_{r}(\lambda)}{I_{0}(\lambda)}=\left|\frac{N_{2}(\lambda)-N_{1}(\lambda)}{N_{2}(\lambda)+N_{1}(\lambda)}\right|^{2},
$$

where $N_{i}$ is the complex refractive index of air or GaAs.

The computed normalized absorption spectra for the nanoparticle-decorated GaAs solar cells are shown in Fig. 5(b). This model calculation well reproduces the experimental results qualitatively, including the enhancements at the band gap edge and the drops at the surface plasmon resonance. The drop extinction in photocurrent for the cells with larger metal nanoparticles is attributed to the significantly higher $\eta_{\text {rad }}$ than that for the smaller nanoparticle cases suppressing the absorption loss in the nanoparticles, also seen in the modeling result. Higher photocurrent enhancement at the band edge for the larger nanoparticle cells than for the smaller cells is reproduced in the modeling, caused mainly by the higher $Q_{e x t}$ for the larger metal nanoparticles.

The $I-V$ curves of the GaAs solar cells under one-sun illumination are given in Fig. 6. The dense arrays of hemispherical nanoparticle give rise to losses in overall short circuit current density, while we have obtained the net photocurrent enhancement in the cells by adjusting the nanoparticle shape and array density. Another advantage of nanoparticle arrays is in apparent in the improvements in the fill factor $(F F)$ for the nanoparticle-decorated cells, indicating the reduction in 
the surface sheet resistance. This effect can be expected in all solar cells that form Ohmic contact between the nanoparticle array and the cell window layer.

(a)

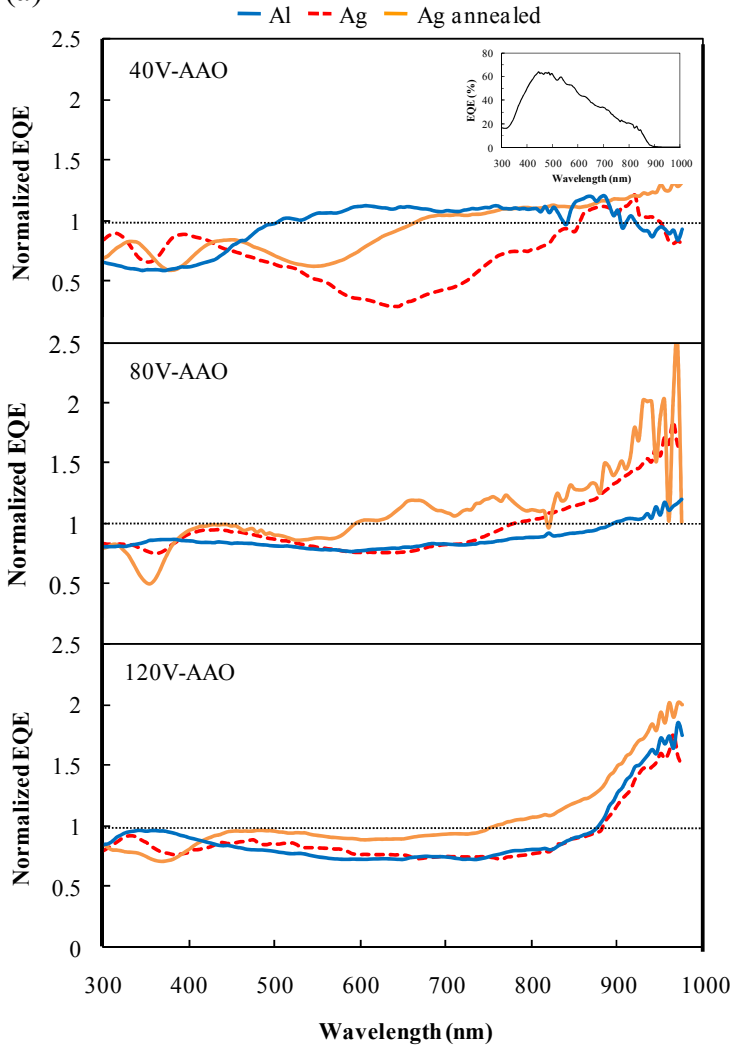

(b)

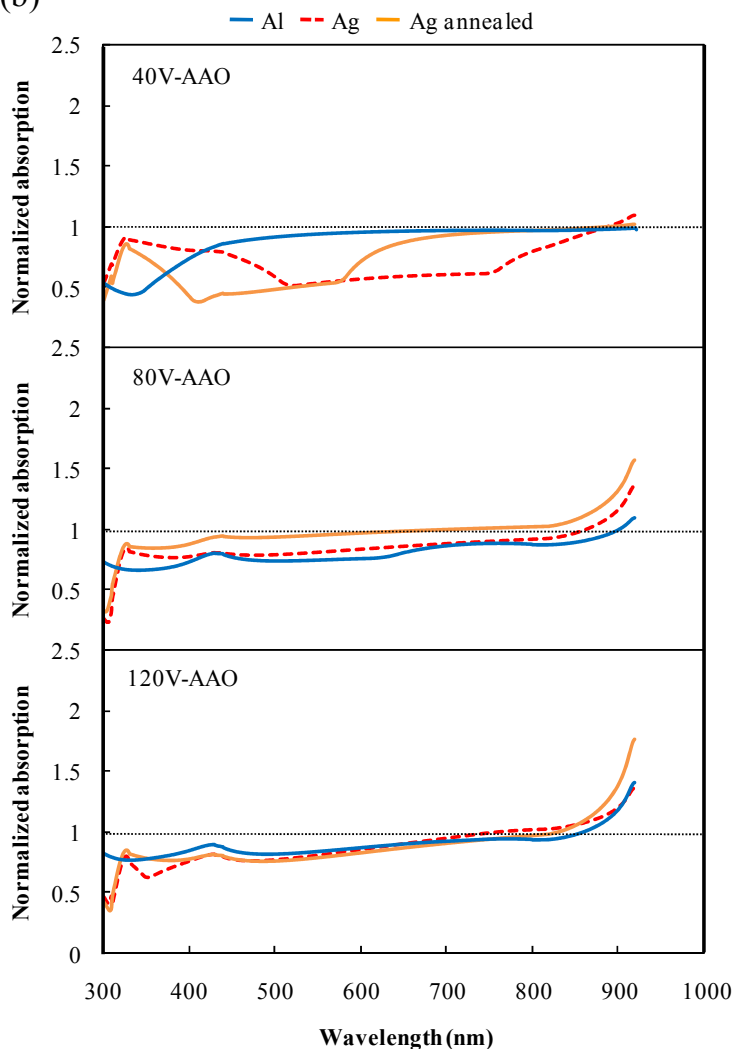

Fig. 5. (a) Photovoltaic spectral responses for the nanoparticle-decorated GaAs solar cells. The spectra are normalized by the spectrum for the reference cell (inset). (b) Calculated absorption spectra for the nanoparticle-decorated GaAs solar cells which are normalized by the calculated absorption spectrum of the reference GaAs cell.

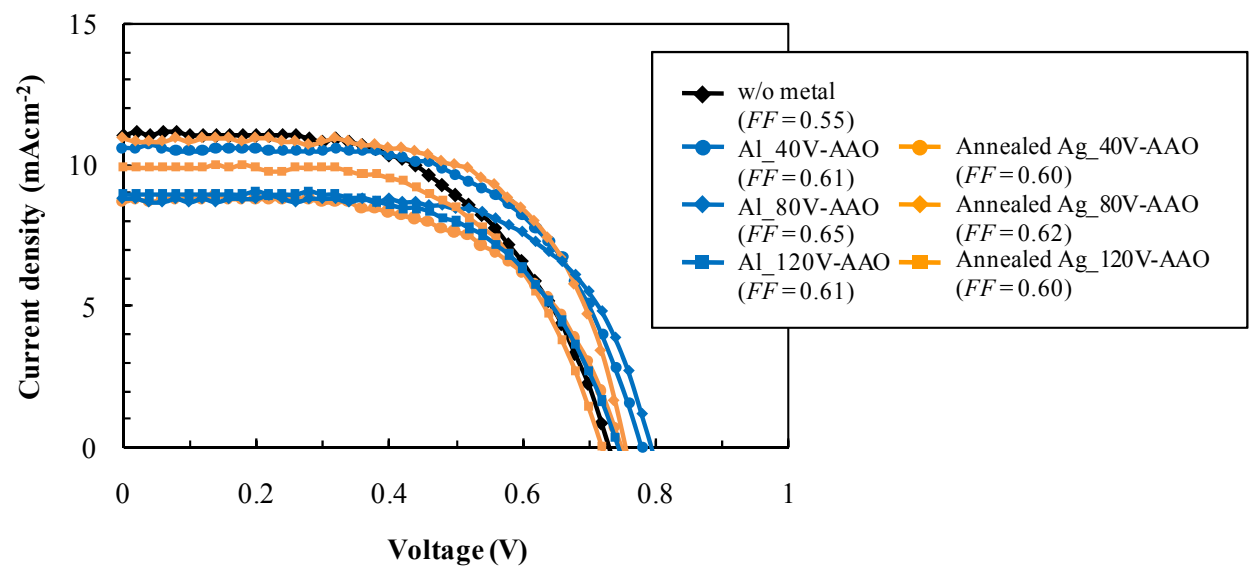

Fig. 6. Photovoltaic $I-V$ curves for the GaAs solar cells under one-sun illumination. 


\section{CONCLUSION}

We have demonstrated the size effect of both $\mathrm{Al}$ and Ag nanoparticles formed on the optically thin GaAs solar cells on the photocurrent enhancements of the cells. At the longer wavelengths than the surface plasmon resonances, scattered incident light by the nanoparticles results in the absorption enhancement in the photovoltaic layers of the cells thus photocurrent enhancement which is maximized near the band gap edge of GaAs. Our results suggest that nanoparticle shape and array density, in addition to nanoparticle size, are important factors in achieving net increased absorption in the solar cell. The GaAs solar cells used in this work consist of GaAs photovoltaic layers on absorbing GaAs substrate, meaning that this structure provide single optical path in the photovoltaic layers. In order to achieve further photocurrent enhancement in the GaAs solar cell, multiple optical path in the photovoltaic layers is needed to enhance the scattering effect by the nanoparticle array, which should be possible by combining plasmonic nanoparticle array with a GaAs solar cell which has greater refractive index contrast between the photovoltaic layers and a low index substrate layer underneath to provide for total internal reflection at the cell back interface and guided wave propagation in the cell photovoltaic layers. ${ }^{[21]}$

\section{ACKNOWLEDGMENTS}

This work was supported by the Department of Energy, Basic Energy Sciences under grant DE-FG02-07ER46405, and Nippon Oil Corporation. We also acknowledge support from the Center for Science and Engineering of Materials (CSEM), an NSF Material Research Science and Engineering Center at Caltech, for facilities use.

\section{REFERENCES}

[1] Stuart, H. R. and Hall, D. G., “Absorption enhancements in silicon-on-insulator waveguides using metal island films," Appl. Phys. Lett. 69, 2327-2329 (1996).

[2] Stuart, H. R. and Hall, D. G., "Island size effects in nanoparticle-enhanced photodetectors," Appl. Phys. Lett. 73, 3815-3817 (1998).

[3] Schaadt, D. M., Feng, B. and Yu, E. T., "Enhanced semiconductor optical absorption via surface plasmon excitation in metal nanoparticles," Appl. Phys. Lett. 86, 063106 (2005).

[4] Pillai, S., Catchpole, K. R., Trupke, T. and Green, M. A., "Surface plasmon enhanced silicon solar cells," J. Appl. Phys. 101, 093105 (2007).

[5] Rand, B. P., Peumans, P. and Forrest, S. R., "Long-range absorption enhancement in organic tandem thin-film solar cells containing silver nanoclusters," J. Appl. Phys. 96, 7519-7526 (2004).

[6] Bohren, C. F. and Huffman, D. R., [Absorption and Scattering of Light by Small Particles], Wiley, New York (1983).

[7] Masuda, H. and Satoh, M., "Fabrication of gold nanodot array using anodic porous alumina as an evaporation mask,” Jpn. J. Appl. Phys. 35, L126-L129 (1996).

[8] Hoyer, P., "Formation of a Titanium Dioxide Nanotube Array," Langmuir 12, 1411-1413 (1996).

[9] Liang, J., Chik, H., Yin, A. and Xu, J., "Two-dimensional lateral superlattices of nanostructures: Nonlithographic formation by anodic membrane template," J. Appl. Phys. 91, 2544-2546 (2002).

[10] Steinhart, M., Wendorff, J. H., Greiner, A., Wehrspohn, R. B., Nielsch, K., Schilling, J., Choi, J. and Gösele, U., "Polymer nanotubes by wetting of ordered porous templates," Science 296, 1997 (2002).

[11] Tobin, S. P., Vernon, S. M., Bajgar, C., Geoffroy, L. M., Keavney, C. J., Sanfacon, M. M. and Haven, V. E., "Device processing and analysis of high efficiency GaAs cells," Solar Cells 24, 103-115 (1988).

[12] Aspnes, D. E., Keiso, S. M., Logan, R. A. and Bhat, R., "Optical properties of $\mathrm{Al}_{x} \mathrm{Ga}_{1-x}$ As," J. Appl. Phys. 60, 754767 (1986).

[13] Kim, J. -H., Lim, D. H. and Yang, G. M., "Selective etching of AlGaAs/GaAs structures using the solutions of citric acid $/ \mathrm{H}_{2} \mathrm{O}_{2}$ and de-ionized $\mathrm{H}_{2} \mathrm{O}$ /buffered oxide etch," J. Vac. Sci. Tech. B 16, 558-560 (1998).

[14] Ono, S., Saito, M. and Asoh, H., "Self-ordering of anodic porous alumina formed in organic acid electrolytes," Electrochim. Acta 51, 827-833 (2005). 
[15] Royer, P., Goudonnet, J. P., Warmack, R. J. and Ferrell, T. L., "Substrate effects on surface-plasmon spectra in metal-island films," Phys. Rev. B 35, 3753-3759 (1987).

[16] Fuster, G., Tyler, J. M., Brener, N. E., Callaway, J. and Bagayoko, D., "Electronic structure and related properties of silver," Phys. Rev. B 42, $7322-7329$ (1990).

[17] Biteen, J. S., Lewis, N. S. and Atwater, H. A., "Spectral tuning of plasmon-enhanced silicon quantum dot luminescence," Appl. Phys. Lett. 88, 131109 (2006).

[18] Palik, E.D. ed., [Handbook of Optical Constants of Solids], Academic Press, Orland (1985).

[19] Jeurgens, L. P. H., Sloof, W. G., Tichelaar, F. D., Borsboom, C. G. and Mittemeijer, E. J., "Determination of thickness and composition of aluminum-oxide overlayers on aluminum substrates," Appl. Surf. Sci. 144-145, 11-15 (1999).

[20] Zhu, W., Hirschmugl, C. J., Laine, A. D. and Sinkovic, B., "Determination of the thickness of Al oxide used as barriers in magnetic tunneling junctions," Appl. Phys. Lett. 78, 3103-3105 (2001).

[21] Tanabe, K., Nakayama, K. and Atwater, H. A. "Plasmon-enhanced absorption and photocurrent in ultrathin GaAs solar cells with metallic nanostructures," to be published in Proceedings of 33rd IEEE Photovoltaic Specialists Conference, IEEE, New York. 